Research Article | Internal Medicine

\title{
Features of Changes in the Structural and Functional State of the Myocardium in Patients with Acute Myocardial Infarction Depending on Body Mass Index Considering FABP4 and CTRP3 Levels
}

\author{
Mariia Koteliukh (D)
}

\begin{abstract}
Introduction. Adipokines such as fatty acid-binding protein 4 (FABP4) and C1q tumor necrosis factor-related protein 3 (CTRP3) can affect the structural and functional state of the myocardium in patients with acute myocardial infarction and obesity.

The objective of the research was to determine the relationship between FABP4, CTRP3 and echocardiographic parameters of the left ventricular myocardium in patients with acute myocardial infarction depending on body mass index.

Materials and Methods. The observational cross-sectional study examined 189 patients with acute myocardial infarction depending on body mass index, who were divided into the following groups: Group 1 included 60 patients with acute myocardial infarction and normal body mass index; Group 2 comprised 68 patients with acute myocardial infarction and excess body weight; Group 3 included 61 patients with acute myocardial infarction and obesity.

Results. In Group 1, the statistical significance correlations were found: between FABP4 and end-diastolic dimension (EDD; $r=-0.458$ ), end-systolic dimension (ESD; $r=-0.460$ ), end-diastolic volume (EDV; $r=-0.452$ ), left ventricular myocardial mass (LVMM; $r=-0.411)$, LVMM/body surface area index (LVMMI2; $r=-0.419$ ); between CTRP3 and EDV $(r=0.425)$, EDD $(r=0.469)$, left ventricular relative posterior wall thickness (LVRPWT; $r=-0.469)$. In Group 2, there were found the statistical significance relationships between: FABP4 and EDD $(r=0.461)$, ESD $(r=0.467)$, EDV $(r=0.449)$, end-systolic volume (ESV; $r=0.485)$, LVMM $(r=0.487)$, LVMMl1 $(r=0.406)$; between CTRP3 and EDD $(r=-0.440)$, EDV $(r=-0.413)$, LVMM $(r=-0.430)$, LVMM/height ${ }^{2.7}$ index (LVMMI1; $r=-0.483$ ). In Group 3, the statistical significance correlations were found between: FABP4 and EDV $(r=0.481)$, ESD $(r=0.411)$, ESV $(r=0.490)$, LVMMI1 $(r=0.403)$; between CTRP3 and EDV $(r=-0.326)$, ESD $(r=-0.367)$, ESV $(r=-0.453)$, LVMMI1 $(r=-0.415)$.

Conclusions. In patients with acute myocardial infarction and overweight/obesity, echocardiographic parameters had a significant low positive correlation with FABP4 and a low negative correlation with CTRP3. On the contrary, in patients with acute myocardial infarction and normal body mass index, echocardiographic parameters had a significant low negative correlation with FABP4 and a low positive correlation with CTRP3.
\end{abstract}

\section{Keywords}

Body Mass Index; CTRP3; FABP4; Echocardiographic Indicators; Myocardial Infarction; Obesity

Department of Internal Medicine No.2, Clinical Immunology and Allergology named after Academician L. T. Malaya, Kharkiv National Medical University, Kharkiv, Ukraine

*E-mail: koteliukh@gmail.com 


\section{Introduction}

Obesity is known to be a risk factor for cardiovascular disease (CVD). Adipose tissue produces pro-inflammatory and anti-inflammatory adipokines. Fatty acid-binding protein 4 (FABP4) and C1q tumor necrosis factor-related protein 3 (CTRP3) are new adipokines that affect carbohydrate and lipid metabolism, inflammation and the development of CVD and their complications $[2,16]$.

FABP4 may be a predictor of acute myocardial infarction (AMI) development [14], and the mechanism of FABP4 action occurs through a number of inflammatory signaling pathways, namely IKK-NF- $\kappa$ B and JNK-AP-1 [4]. According to a study conducted by Obokata M et al. [10], FABP4 is released from adipocytes through $\beta 3$-AR-mediated lipolysis, which may accompany the development of AMI. According to Aleksandrova $\mathrm{K}$ et al. [1] obesity had a significant effect on FABP4 levels in the presence of AMI. Systolic and diastolic function was shown to be reduced in subjects with high concentrations of serum FABP4 [11]. FABP4 can affect cardiac function through its direct action on cardiomyocytes [8]. Therefore, a causal relationship between FABP4 and cardiomyocyte physiology was determined. FABP4 can affect cardiac function in a paracrine or endocrine manner.

Yuan Yu-Pei et al. [19] have determined that CTRP3 is produced not only in adipocytes, but also in the heart and the mechanism of CTRP3 action on the myocardium is carried out through Akt, AMPK, NF\&kappa; B signaling pathways. CTRP3 is an anti-inflammatory adipokine that improves insulin sensitivity and reduces hepatocyte gluconeogenesis [18], has cardioprotective properties and reduces myocardial damage after ischemia [13], weakens pathological myocardial reconstruction after acute myocardial infarction due to inhibition of myocardial fibrosis and improves the survival and regeneration of ischemic cardiomyocytes [17]. Endogenous CTRP3 plays a significant role in the regulation of cardiac remodeling after AMI, and recombinant CTRP3 may have therapeutic potential in the treatment of AMI [20]. However, the effect of FABP4 and CTRP3 on the structural and functional state of the myocardium in patients with AMI depending on body mass index (BMI) has not been studied so far.

The objective of the research was to determine the relationship between FABP4, CTRP3 and echocardiographic parameters of the left ventricular (LV) myocardium in patients with AMI depending on BMI.

\section{Materials and Methods}

\section{Study Design}

In this observational cross-sectional study, we enrolled patients with ST-segment elevation AMI who were hospitalized to the intensive care units of State Institution " $L$. T. Malaya National Institute of Therapy of the National Academy of Medical Sciences of Ukraine" and Kharkiv Clinical Hospital of Rail Transport No. 1 of Branch "Health Center" of the Public
Joint Stock Company "Ukrainian Railways". The study was performed between September 01, 2018 and December 31, 2020.

We followed the 2017 European Society of Cardiology guidelines for the diagnosis and management of patients with AMI with ST-segment elevation [6]. All patients underwent stenting of the affected coronary artery and subsequently received the following drug therapy: anticoagulant, acetylsalicylic acid, ticagrelor or clopidogrel, high dose of statin, nitrates, $\beta$-blocker (depending on heart rate), and angiotensinconverting enzyme inhibitor (for blood pressure correction). Serum collection and echocardiographic examination were performed within 1-2 days of follow-up.

\section{Study Groups}

The study involved 189 patients with AMI depending on BMI: Group 1 included 60 patients with AMI and normal BMI; Group 2 comprised 68 patients with AMI and excess body weight (EBW); Group 3 amounted for 61 patients with AMI and obesity.

Exclusion criteria were type 1 and 2 diabetes mellitus, Covid 19, autoimmune diseases, diffuse connective tissue diseases, pituitary and hypothalamic diseases, thyroid disease, symptomatic hypertension, valvular heart disease, chronic heart failure (CHF) of IV functional class (FC), chronic obstructive pulmonary disease, severe liver and kidney dysfunction, severe anemia, cancer.

The groups were comparable by baseline characteristics, which is presented in Table 1.

\section{Blood Analysis and Body Measurements}

All studies were conducted in the Biochemical Department of the Central Research Laboratory of Kharkiv National Medical University of the Ministry of Health of Ukraine. Patients' serum samples were stored at $-80^{\circ} \mathrm{C}$ until the study. Serum concentration of FABP4 and CTRP 3 in patients was determined by enzyme-linked immunosorbent assay on Labline90 analyzer (Austria) using commercial test systems Human FABP4 (Elabscience Biotechnology, USA) and Human CTRP3 (Aviscera Bioscience Inc, Santa Clara, USA) according to the manufacturer's instructions. The method for determining the content of FABP4 and CTRP 3 is based on sandwich technology, which is characterized by double binding of biotin-containing antibodies to the test substance.

The hospital laboratory performed a set of routine tests. Blood samples were taken within 1-2 days. Serum FABP4 and CTRP3 were determined in nanograms per milliliter of serum $(\mathrm{ng} / \mathrm{ml})$.

Serum troponin I was determined by enzyme-linked immunosorbent assay (reference ranges $-<0.05 \mathrm{ng} / \mathrm{ml}$ for men and women).

To determine the optimal weight, BMI (the Quetelet index) was used, which was calculated as a patient's weight in kilograms divided by the square of the patient's height in meters. To determine the type of adipose tissue distribution, 
Table 1. General characteristics of the groups under study.

\begin{tabular}{|c|c|c|c|c|c|c|}
\hline \multirow[t]{2}{*}{ Indicator, unit of measurement } & $\begin{array}{c}\text { Patients with AMI and } \\
\text { normal BMI }\end{array}$ & $\begin{array}{c}\text { Patients with AMI and } \\
\text { EBW }\end{array}$ & $\begin{array}{c}\text { Patients with AMI and } \\
\text { obesity }\end{array}$ & \multirow{2}{*}{\multicolumn{3}{|c|}{ Probability $(\mathrm{p})$}} \\
\hline & 1 & 2 & 3 & & & \\
\hline Age, years & $58.42 \pm 8.25$ & $60.03 \pm 6.61$ & $57.93 \pm 8.63$ & \multicolumn{3}{|c|}{$\mathrm{p}=0.9$} \\
\hline Women, n (\%) & $5(8.33)$ & $11(16)$ & $18(30)$ & \multicolumn{3}{|c|}{$\mathrm{p}=0.03$} \\
\hline Men, n $(\%)$ & $55(91.67)$ & $57(84)$ & $43(70)$ & \multicolumn{3}{|c|}{$\mathrm{p}=0.39$} \\
\hline \multicolumn{7}{|l|}{ In history: } \\
\hline Stable angina pectoris & $20(33)$ & $22(32)$ & $25(41)$ & \multicolumn{3}{|c|}{$\mathrm{p}=0.679$} \\
\hline Post-infarction cardiosclerosis & $5(8)$ & $5(7)$ & $5(8)$ & \multicolumn{3}{|c|}{$\mathrm{p}=0.880$} \\
\hline Arterial hypertension & $55(92)$ & $60(88)$ & $57(93)$ & \multicolumn{3}{|c|}{$\mathrm{p}=0.829$} \\
\hline Atrial fibrillation & $5(8)$ & $5(7)$ & $5(8)$ & \multicolumn{3}{|c|}{$\mathrm{p}=0.880$} \\
\hline \multicolumn{7}{|l|}{ CHF, FC, n (\%): } \\
\hline I & $35(58.33)$ & $47(69)$ & $29(48)$ & \multicolumn{3}{|c|}{$\mathrm{p}=0.10$} \\
\hline II & $8(13.33)$ & $9(13)$ & $8(13)$ & \multicolumn{3}{|c|}{$\mathrm{p}=0.89$} \\
\hline III & $17(28.33)$ & $12(18)$ & $24(39)$ & \multicolumn{3}{|c|}{$\mathrm{p}=0.17$} \\
\hline \multirow{3}{*}{ BMI, kg/m² } & \multirow{3}{*}{$23.52 \pm 0.16$} & \multirow{3}{*}{$27.06 \pm 0.15$} & \multirow{3}{*}{$32.77 \pm 2.47$} & & 2 & 3 \\
\hline & & & & 1 & $<0.001$ & $<0.001$ \\
\hline & & & & 2 & - & $<0.001$ \\
\hline \multirow{3}{*}{ Height, $\mathrm{cm}$} & \multirow{3}{*}{$173.0 \pm 6.92$} & \multirow{3}{*}{$175.4 \pm 7.21$} & \multirow{3}{*}{$169.85 \pm 8.05$} & & 2 & 3 \\
\hline & & & & 1 & 0.06 & 0.023 \\
\hline & & & & 2 & - & $<0.001$ \\
\hline \multirow{3}{*}{ Weight, kg } & \multirow{3}{*}{$70.93 \pm 7.63$} & \multirow{3}{*}{$83.58 \pm 7.38$} & \multirow{3}{*}{$94.88 \pm 11.44$} & & 2 & 3 \\
\hline & & & & 1 & $<0.001$ & $<0.001$ \\
\hline & & & & 2 & - & $<0.001$ \\
\hline \multirow{3}{*}{$\mathrm{WC}, \mathrm{cm}$} & \multirow{3}{*}{$77.07 \pm 7.67$} & & & & 2 & 3 \\
\hline & & $89.65 \pm 6.8$ & $129.53 \pm 31.53$ & 1 & $<0.001$ & $<0.001$ \\
\hline & & & & 2 & - & $<0.001$ \\
\hline & & & & & 2 & 3 \\
\hline $\mathrm{TC}, \mathrm{cm}$ & $100.63 \pm 6.01$ & $108.17 \pm 4.7$ & $109.17 \pm 8.23$ & 1 & $<0.001$ & $<0.001$ \\
\hline & & & & 2 & - & 0.394 \\
\hline & & & & & 2 & 3 \\
\hline WC/TC & $0.76 \pm 0.06$ & $0.84 \pm 0.05$ & $1.21 \pm 0.34$ & 1 & $<0.001$ & $<0.001$ \\
\hline & & & & 2 & - & $<0.001$ \\
\hline & & & & & 2 & 3 \\
\hline Troponin I, ng/ml & $2.03 \pm 1.98$ & $2.34 \pm 2.98$ & $3.41 \pm 2.29$ & 1 & 0.602 & 0.006 \\
\hline & & & & 2 & - & 0.056 \\
\hline
\end{tabular}

Notes: $\mathrm{BMI}$ - body mass index; WC - waist circumference; TC - thigh circumference; WC/TC - WC to TC ratio; $\mathrm{CHF}$ - chronic heart failure; FC - functional class.

the waist-to-thigh circumference ratio (WC/TC) was calculated. Waist circumference (WC) was measured as the smallest circumference below the thorax and above two to three centimeters $(\mathrm{cm})$ above the navel; thigh circumference (TC) is measured at the level of 5-7 cm below the gluteal fold and is the widest part of the thighs. The values of anthropometric indicators are shown in Table 2.

Cardiometabolic risk increases in $\mathrm{WC}$ values $>102.0 \mathrm{~cm}$ for men and $>88.0 \mathrm{~cm}$ for women. The value of the WC/TC ratio $\geq 0.90$ for men and $\geq 0.85$ for women is a sign of abdominal obesity [12].

\section{Instrumental Procedures}

Doppler echocardiography was performed according to the conventional method on an ultrasound scanner Radmir ULTIMA Pro30. The study implied determination
Table 2. Characteristics of anthropometric indicators.

\begin{tabular}{lcc}
\hline BMI value, $\mathrm{kg} / \mathrm{m}^{2}$ & Value \\
\hline Normal BMI & & $18.5-24.9$ \\
EBW & & $25.0-29.9$ \\
Obesity & $\geq 30.0$ \\
\multirow{2}{*}{ WC } & Male & $<94.0 \mathrm{~cm}$ \\
& Female & $<80.0 \mathrm{~cm}$ \\
\hline \multirow{2}{*}{ WC/TC } & Male & $<0.90$ \\
& Female & $<0.85$ \\
\hline
\end{tabular}

of LV end-diastolic dimension (EDD) and end-systolic dimension (ESD), interventricular septal thickness (IVST), aortic dimension, left atrial (LA) dimension, and left ventricular posterior wall thickness (LVPWT) during diastole. LV 
myocardial mass (LVMM), the LVMM index (LVMMI) 1 $\left(\right.$ LVMMI1 $=$ LVMM/height $\left(\mathrm{m}^{2.7}\right)$, which is appropriate in EBW and obesity), the LVMMI2 (LVMMI2 = LVMM/body surface area $\left(\mathrm{m}^{2}\right)$ ) were calculated. When using the LVMMI1 in the diagnosis of LV hypertrophy (LVH), the upper limit of the norm was considered as $44 \mathrm{~g} / \mathrm{m}^{2.7}$ for women and $48 \mathrm{~g} / \mathrm{m}^{2.7}$ for men. LVH was defined at an LVMMI2 value of more than $110 \mathrm{~g} / \mathrm{m}^{2}$ for women and more than $125 \mathrm{~g} / \mathrm{m}^{2}$ for men. Left ventricular relative wall thickness (LVRWT) $($ LVRWT $=($ LVPWT + IVST $) /$ EDD $))$, the LVMMI1 and LVMMI2 indicators were used to determine the type of LV remodeling. Concentric LV remodeling was determined in LVRPWT $\geq 0.45$ and normal LVMMI $[3,9]$.

\section{Statistical Analysis}

Statistical data processing was performed using the licensed software package IBM SPPS Statistics 27.0 (IBM Inc., USA, license No. L-CZAA-BKKMKE). The hypothesis of normal distribution of indicators was tested by the KolmogorovSmirnov test. Basic statistical parameters were calculated for interval scale variables (mean, SD). Nominal variables were expressed as number and percentage. Paired comparison was rendered using the $t$ test. Nominal variables were compared using the $\chi^{2}$ test with Yates's correction, depending on the size of the groups. Evaluation of the significance of the difference between the means of multiple comparisons for quantitative traits with normal distribution using the Bonferroni correction was performed by one-way analysis of variance (ANOVA). Parameter relationships were analyzed using the Pearson correlation coefficient ( $r$ ). The difference was considered significant at $\mathrm{p}<0.05$ values.

\section{Results}

Table 3 shows the echocardiographic parameters in the study groups. Significant differences were found between echocardiographic parameters in patients with AMI and obesity (Group 3) due to an increase in ESD, end-diastolic volume (EDV), end-systolic volume (ESV), LA dimension, LVMM, LVMMI1 as compared to patients with AMI and normal BMI (Group 1) $(\mathrm{p}<0.05)$.

The results obtained showed that patients with AMI and obesity as compared to patients with AMI and normal BMI had a significant increase in echocardiographic parameters, namely: EDV by $16.49 \%$; ESV by $30.19 \%$; ESD by $11.45 \%$; LA dimension by $11.83 \%$; LVMMI1 by $20.76 \%$; LVMM by $16.81 \%$.

Patients with AMI and EBW (Group 2) were not found to have any significant difference between echocardiographic parameters as compared to patients with AMI and normal BMI (Group 1), except for LA dimension ( $\mathrm{p}<0.001)$. Comparison of the second and third groups showed a significant difference in the LVMMI1 $(\mathrm{p}<0.001)$.

The values of FABP4 and CTRP3 levels in patients with AMI depending on BMI are shown in Table 4. There was a significant difference in the content of FABP4 and CTRP 3 in obese patients as compared to patients with AMI and normal BMI $(\mathrm{p}<0.001)$. Patients with AMI and EBW had low CTRP3 concentrations as compared to patients with AMI and normal BMI $(\mathrm{p}<0.001)$.

The correlations between anthropometric parameters and adipokines in AMI patients with normal BMI are shown in Table 5A.

Table 5B shows the correlations between anthropometric parameters and adipokines in patients with AMI and EBW.

The relationship between anthropometric parameters and adipokines in obese patients with AMI is shown in Table 5C.

Patients with AMI, regardless of BMI values, were found to have a positive correlation between troponin I and FABP4 $(\mathrm{r}=0.457, \mathrm{p}=0.009)$ and inverse correlation with CTRP3 $(\mathrm{r}=-0.415, \mathrm{p}=0.008)$.

Patients with AMI and normal BMI were shown to have an inversely low correlation between FABP4, EDD, ESD, EDV, LVMM, LVMMI1, LVMMI2 and a positive low correlation between CTRP3, EDD, EDV, LVRPWT (Table 6).

Patients with AMI and EBW had a direct low correlation between FABP4, EDD, ESD, EDV, ESV, LVMM, LVMMI1, LVMMI2, and an inverse low correlation between CTRP3, EDD, EDV, SV, LVMM, LVMMI1, LVMMI2 (Table 7).

Patients with AMI and obesity had a direct low relationship between FABP4, EDV, ESD, ESV, LVMMI1, and a low inverse correlation between CTRP3, EDV, ESD, ESV, LVMMI1 (Table 8).

\section{Discussion}

According to Obokata M et al. [10], FABP4 levels peaked on admission to the hospital or immediately after percutaneous coronary intervention in patients with AMI. Patients with AMI, normal BMI and EBW were shown to have inverse moderate relationships between FABP4, BMI and WC/TC. We found a direct correlation between FABP4, BMI and WC/TC in patients with AMI and obesity. Aleksandrova $\mathrm{K}$ et al. [1] has determined a direct correlation between the concentration of FABP4, BMI and WC and noted that a high concentration of FABP4 was associated with an increase in BMI and WC.

Von Jeinsen B et al. [15] have determined a positive correlation between the content of FABP4 and cardiac troponin T and reported the low LVMMI, slightly reduced LVEDD and an inverse correlation between the LVMMI, LVEDD and FABP4 levels in patients with coronary heart disease. Harada T et al. [5] have indicated increased LVMMI and determined a direct relationship between FABP4 and LVMMI levels, LA volume index, LV stiffness. In our study, FABP4 content was associated with an increase in myocardial mass and dilation of the heart cavities due to increased EDV, ESV, ESD, LVMMI1 and, thus, contributed to the deterioration of the structural and functional state of the myocardium in patients with AMI and obesity.

Yi W et al. [18] have found a low concentration of CTRP3 on day 1 of AMI; the lowest level of CTRP3 was determined 3 days after AMI. Choi Kyung Mook et al. [7] have found that 
Table 3. Echocardiographic parameters in different groups of patients.

\begin{tabular}{|c|c|c|c|c|c|c|}
\hline \multirow[t]{2}{*}{ Indicator, unit of measurement } & $\begin{array}{c}\text { Patients with AMI } \\
\text { and normal BMI }\end{array}$ & $\begin{array}{c}\text { Patients with AMI } \\
\text { and EBW }\end{array}$ & $\begin{array}{l}\text { Patients with AMI } \\
\text { and obesity }\end{array}$ & \multirow{2}{*}{\multicolumn{3}{|c|}{ Probability (p) }} \\
\hline & & & 3 & & & \\
\hline \multirow{3}{*}{$\mathrm{EDD}, \mathrm{cm}$} & & & & & 2 & 3 \\
\hline & $4.90 \pm 0.50$ & $5.0 \pm 0.52$ & $5.14 \pm 0.61$ & 1 & 0.262 & 0.05 \\
\hline & & & & 2 & - & 0.185 \\
\hline \multirow{3}{*}{$\mathrm{ESD}, \mathrm{cm}$} & & & & & 2 & 3 \\
\hline & $3.58 \pm 0.58$ & $3.60 \pm 0.68$ & $3.99 \pm 0.99$ & 1 & 0.82 & 0.011 \\
\hline & & & & 2 & - & 0.076 \\
\hline \multirow{3}{*}{$\mathrm{EDV}, \mathrm{ml}$} & & & & & 2 & 3 \\
\hline & $117.8 \pm 26.53$ & $121.5 \pm 31.51$ & $137.22 \pm 39.59$ & 1 & 0.477 & 0.005 \\
\hline & & & & 2 & - & 0.05 \\
\hline \multirow{3}{*}{$\mathrm{ESV}, \mathrm{ml}$} & & & & & 2 & 3 \\
\hline & $53.92 \pm 20.05$ & $58.64 \pm 21.51$ & $70.20 \pm 30.41$ & 1 & 0.675 & 0.001 \\
\hline & & & & 2 & - & 0.092 \\
\hline \multirow{3}{*}{$\mathrm{SV}, \mathrm{ml}$} & & & & & 2 & 3 \\
\hline & $64.03 \pm 14.54$ & $65.81 \pm 19.09$ & $68.92 \pm 20.34$ & 1 & 0.39 & 0.218 \\
\hline & & & & 2 & - & 0.383 \\
\hline \multirow{3}{*}{$\mathrm{EF}, \%$} & & & & & 2 & 3 \\
\hline & $53.90 \pm 9.08$ & $53.38 \pm 8.59$ & $50.35 \pm 9.83$ & 1 & 0.737 & 0.111 \\
\hline & & & & 2 & - & 0.087 \\
\hline \multirow{3}{*}{ IVST, cm } & & & & & 2 & 3 \\
\hline & $1.16 \pm 0.23$ & $1.21 \pm 0.18$ & $1.21 \pm 0.16$ & 1 & 0.614 & 0.335 \\
\hline & & & & 2 & - & 0.805 \\
\hline \multirow{3}{*}{ LVPWT, cm } & & & & & 2 & 3 \\
\hline & $1.17 \pm 0.20$ & $1.19 \pm 0.19$ & $1.22 \pm 0.15$ & 1 & 0.59 & 0.32 \\
\hline & & & & 2 & - & 0.202 \\
\hline \multirow{3}{*}{$\mathrm{LA}, \mathrm{cm}$} & & & & & 2 & 3 \\
\hline & $3.38 \pm 0.51$ & $3.74 \pm 0.55$ & $3.78 \pm 0.62$ & 1 & $<0.001$ & $<0.001$ \\
\hline & & & & 2 & - & 0.644 \\
\hline \multirow{3}{*}{ Aorta, cm } & & & & & 2 & 3 \\
\hline & $3.11 \pm 0.46$ & $3.08 \pm 0.57$ & $3.20 \pm 0.47$ & 1 & 0.759 & 0.453 \\
\hline & & & & 2 & - & 0.058 \\
\hline \multirow{3}{*}{ LVMM, g } & & & & & 2 & 3 \\
\hline & $264.61 \pm 74.71$ & $281.8 \pm 71.22$ & $309.1 \pm 83.33$ & 1 & 0.154 & 0.006 \\
\hline & & & & 2 & - & 0.085 \\
\hline \multirow{3}{*}{ LVMMI1, g/m } & & & & & 2 & 3 \\
\hline & $51.3 \pm 13.63$ & $52.27 \pm 12.32$ & $61.95 \pm 15.44$ & 1 & 0.723 & $<0.001$ \\
\hline & & & & 2 & - & $<0.001$ \\
\hline \multirow{3}{*}{ LVMMI $2, \mathrm{~g} / \mathrm{m}^{2}$} & & & & & 2 & 3 \\
\hline & $144.36 \pm 39.83$ & $144.59 \pm 35.11$ & $143.80 \pm 37.38$ & 1 & 0.97 & 0.858 \\
\hline & & & & 2 & - & 0.926 \\
\hline \multirow{3}{*}{ LVRPWT } & & & & & 2 & 3 \\
\hline & $0.48 \pm 0.09$ & $0.48 \pm 0.09$ & $0.48 \pm 0.07$ & 1 & 0.842 & 0.882 \\
\hline & & & & 2 & & 0.611 \\
\hline Hypokinesia & $48(80)$ & $58(85)$ & $48(79)$ & \multicolumn{3}{|c|}{$\mathrm{p}=0.721$} \\
\hline Akinesia & $12(20)$ & $10(15)$ & $13(21)$ & & $\mathrm{p}=0.7$ & \\
\hline
\end{tabular}


Table 4. Determination of FABP4 and CTRP3 levels in AMI patients depending on BMI.

\begin{tabular}{|c|c|c|c|c|c|c|}
\hline \multicolumn{7}{|c|}{ BMI } \\
\hline Indicator & $18.5-24.9$ & $25-29.9$ & $>30.0$ & & \multirow{2}{*}{\multicolumn{2}{|c|}{$\mathrm{p}$}} \\
\hline & 1 & 2 & 3 & & & \\
\hline \multirow{3}{*}{ FABP 4} & & & & & 2 & 3 \\
\hline & $9.76 \pm 2.07$ & $10.93 \pm 2.6$ & $11.97 \pm 4.21$ & 1 & 0.098 & $<0.001$ \\
\hline & & & & 2 & - & 0.17 \\
\hline \multirow{3}{*}{ CTRP 3} & & & & & 2 & 3 \\
\hline & $272.31 \pm 56.98$ & $235.69 \pm 49.49$ & $234.03 \pm 43.17$ & 1 & $<0.001$ & $<0.001$ \\
\hline & & & & 2 & - & 0.82 \\
\hline
\end{tabular}

lower levels of CTRP3 were associated with higher weight and WC. Patients with AMI, normal BMI and EBW were found to have positive moderate correlations between CTRP3, BMI and WC. We found an inverse relationship between CTRP3, BMI and WC in patients with AMI and obesity and an inverse relationship between CTRP3 and EDV, ESV, ESD, LVMMI1, which is fully consistent with data on the anti-inflammatory properties of CTRP3 [13]. According to a study conducted by Yi W et al. [18], administration of recombinant CTRP3 led to a decrease in the size and weight of the heart, maintenance of EDD, ESD of the LV in AMI.

Our study showed a tendency to an increase in the concentration of FABP4 and a decrease in the content of CTRP3 in patients with AMI depending on BMI. It should be noted that a positive low correlation between troponin I and FABP4 levels and an inverse low correlation between troponin I and CTRP3 concentration were found in all patients with AMI. We found that FABP4 and CTRP3 were associated with anthropometric indicators. In our opinion, the imbalance in the adipokine system due to changes in FABP4 and CTRP3 levels is associated not only with the presence of EBW and obesity, but also with AMI development. Decreased serum CTRP3 concentration and increased FABP4 levels result in structural and functional changes in the LV myocardium in the form of increased cavities and dimension of the left ventricle in the comorbid patient.

\section{Conclusions}

An increase in FABP4 concentration and a decrease in CTRP3 levels were associated with an increase in BMI, WC and WC/TC.

Anthropometric indicators that characterize EBW had a significant moderate positive correlation with FABP4 and a low negative correlation with CTRP.

Echocardiographic indicators in patients with AMI and normal BMI had a significant low negative correlation with FABP4 and a low positive correlation with CTRP3.
Increased FABP4 levels and decreased CTRP3 concentration were associated with increased LV myocardial cavities and dimensions in patients with AMI and overweight obesity.

\section{Ethical Statement}

This study is a fragment of the scientific-research work of the Department of Internal Medicine No. 2, Clinical Immunology and Allergology named after Academician L.T. Malaya of Kharkiv National Medical University - "Prediction of the Clinical Course, Improvement of Diagnosis and Treatment of Ischemic Heart Disease and Arterial Hypertension in Patients with Metabolic Disorders", No. 0120U102025, 2020-2022.

The research was conducted according to the WMA Declaration of Helsinki "Ethical Principles for Medical Research Involving Human Subjects". The design of the study was approved by the Ethics Commission of Kharkiv National Medical University (Minutes No. 2 dated April 02, 2018).

\section{Informed Consent}

All patients who participated in the study signed a voluntary informed consent to participate.

\section{Conflict of Interest}

The authors declare that no conflicts exist.

\section{Financial Disclosure}

The authors declared no financial support. 
Table 5. Matrix of intercorrelations between anthropometric indicators and adipokines.

\begin{tabular}{|c|c|c|c|c|c|c|c|c|c|c|c|c|c|}
\hline \multicolumn{7}{|c|}{ (A) patients with AMI and normal BMI } & \multicolumn{7}{|c|}{ (B) patients with AMI and EBW } \\
\hline Indicator & 1 & 2 & 3 & 4 & 5 & 6 & Indicator & 1 & 2 & 3 & 4 & 5 & 6 \\
\hline 1. FABP4 & 1 & $-0.427 *$ & $-0.571 *$ & -0.238 & -0.231 & $-0.512 *$ & 1. FABP4 & 1 & $-0.481 *$ & $0.617 *$ & 0.274 & 0.217 & $0.517 *$ \\
\hline 2. CTRP3 & & 1 & $0.403 *$ & $0.405^{*}$ & 0.271 & $0.408 *$ & 2. CTRP3 & & 1 & $-0.405^{*}$ & $-0.407 *$ & -0.205 & $-0.348 *$ \\
\hline 3. BMI & & & 1 & $0.519 *$ & $0.479 *$ & 0.259 & 3. BMI & & & 1 & 0.189 & $0.411 *$ & 0.239 \\
\hline 4. WC & & & & 1 & $0.665^{*}$ & $0.776^{*}$ & 4. WC & & & & 1 & $0.538 *$ & $0.576^{*}$ \\
\hline 5. TC & & & & & 1 & 0.161 & 5. TC & & & & & 1 & 0.162 \\
\hline 6. WC/TC & & & & & & 1 & 6. WC/TC & & & & & & 1 \\
\hline
\end{tabular}

\begin{tabular}{|c|c|c|c|c|c|c|}
\hline \multicolumn{7}{|c|}{ (C) patients with AMI and obesity } \\
\hline Indicator & 1 & 2 & 3 & 4 & 5 & 6 \\
\hline 1. FABP4 & 1 & $-0.487 *$ & $0.671 *$ & 0.492 & 0.407 & $0.528 *$ \\
\hline 2. CTRP3 & & 1 & $-0.408 *$ & $-0.408^{*}$ & -0.31 & -0.357 \\
\hline 3. BMI & & & 1 & 0.13 & 0.193 & 0.11 \\
\hline 4. WC & & & & 1 & 0.185 & 0.139 \\
\hline 5. TC & & & & & 1 & 0.168 \\
\hline 6. WC/TC & & & & & & 1 \\
\hline
\end{tabular}

Table 6. Correlation between echocardiographic parameters and adipokines in patients with AMI and normal BMI.

\begin{tabular}{|c|c|c|c|c|c|c|c|c|c|c|c|c|c|c|c|c|}
\hline Indicator & 1 & 2 & 3 & 4 & 5 & 6 & 7 & 8 & 9 & 10 & 11 & 12 & 13 & 14 & 15 & 16 \\
\hline 1. FABP4 & 1 & $-0.493^{*}$ & $-0.458^{* *}$ & $-0.460^{* *}$ & $-0.452^{* *}$ & -0.247 & -0.203 & 0.16 & -0.011 & -0.23 & $-0.487^{* *}$ & -0.176 & $-0.411^{* *}$ & $-0.426^{* *}$ & $-0.419^{* *}$ & 0.153 \\
\hline 2. CTRP3 & & 1 & $0.469^{* *}$ & 0.198 & $0.425^{* *}$ & 0.24 & $0.407^{*}$ & -0.081 & -0.185 & 0.016 & 0.201 & 0.254 & 0.285 & 0.269 & 0.262 & $-0.469^{* *}$ \\
\hline 3. EDD & & & 1 & $0.361^{* *}$ & $0.698^{* *}$ & $0.596^{* *}$ & $0.381^{* *}$ & -0.281 & -0.055 & 0.243 & 0.063 & $0.462^{* *}$ & $0.799^{* *}$ & $0.707^{* *}$ & $0.779^{* *}$ & $-0.632^{* *}$ \\
\hline 4. ESD & & & & 1 & $0.608^{* *}$ & $0.748^{* *}$ & $0.325^{*}$ & $-0.489^{* *}$ & -0.103 & 0.262 & 0.079 & $0.358^{* *}$ & 0.261 & 0.142 & 0.207 & -0.242 \\
\hline 5. EDV & & & & & 1 & $0.764^{* *}$ & $0.548^{* *}$ & $-0.383^{* *}$ & -0.072 & -0.019 & $0.392^{* *}$ & $0.397^{* *}$ & $0.493^{* *}$ & $0.365^{* *}$ & $0.448^{* *}$ & $-0.519^{* *}$ \\
\hline 6. ESV & & & & & & 1 & 0.167 & $-0.731^{* *}$ & -0.05 & 0.051 & $0.415^{* *}$ & $0.382^{* *}$ & $0.460^{* *}$ & $0.367^{* *}$ & $0.378^{* *}$ & $-0.403^{* *}$ \\
\hline 7. SV & & & & & & & 1 & $0.328^{*}$ & -0.237 & -0.161 & 0.288 & 0.288 & 0.135 & -0.031 & 0.13 & $-0.435^{* *}$ \\
\hline 8. $\mathrm{EF}$ & & & & & & & & 1 & -0.161 & -0.129 & -0.233 & -0.131 & $-0.312^{*}$ & $-0.345^{* *}$ & -0.213 & 0.094 \\
\hline 9. IVST & & & & & & & & & 1 & $0.524^{* *}$ & 0.094 & 0.045 & $0.483^{* *}$ & $0.393^{* *}$ & $0.413^{* *}$ & $0.649^{* *}$ \\
\hline 10. LVPWT & & & & & & & & & & 1 & 0.179 & $0.308^{*}$ & $0.698^{* *}$ & $0.631^{* *}$ & $0.643^{* *}$ & $0.478^{* *}$ \\
\hline 11. LA & & & & & & & & & & & 1 & $0.529^{* *}$ & $0.442^{* *}$ & 0.267 & 0.297 & -0.265 \\
\hline 12. Aorta & & & & & & & & & & & & 1 & $0.408^{* *}$ & 0.287 & 0.268 & -0.146 \\
\hline 13. LVMM & & & & & & & & & & & & & 1 & $0.870^{* *}$ & $0.942^{* *}$ & -0.079 \\
\hline 14. LVMMI1 & & & & & & & & & & & & & & 1 & $0.858^{* *}$ & -0.062 \\
\hline 15. LVMMI2 & & & & & & & & & & & & & & & 1 & -0.103 \\
\hline 16. LVRWT & & & & & & & & & & & & & & & & 1 \\
\hline
\end{tabular}

Notes: $*-\mathrm{p}<0.05, * *-\mathrm{p}<0.01$ 
Table 7. Correlation between echocardiographic parameters and adipokines in patients with AMI and EBW.

\begin{tabular}{|c|c|c|c|c|c|c|c|c|c|c|c|c|c|c|c|c|}
\hline Indicator & 1 & 2 & 3 & 4 & 5 & 6 & 7 & 8 & 9 & 10 & 11 & 12 & 13 & 14 & 15 & 16 \\
\hline 1. FABP4 & 1 & $-0.431^{*}$ & $0.461^{* *}$ & $0.467^{* *}$ & $0.449^{* *}$ & $0.485^{* *}$ & 0.296 & $-0.303^{*}$ & 0.115 & 0.18 & $0.415^{* *}$ & 0.15 & $0.487^{* *}$ & $0.406^{* *}$ & $0.414^{* *}$ & -0.113 \\
\hline 2. CTRP3 & & 1 & $-0.440^{*}$ & -0.232 & $-0.413^{*}$ & -0.131 & $-0.435^{* *}$ & 0.062 & -0.159 & -0.058 & 0.047 & -0.108 & $-0.430^{* *}$ & $-0.483^{* *}$ & $-0.484^{* *}$ & -0.145 \\
\hline 3. EDD & & & 1 & $0.597^{* *}$ & $0.960^{* *}$ & $0.667^{* *}$ & $0.751^{* *}$ & -0.278 & -0.253 & -0.1 & -0.054 & 0.25 & $0.571^{* *}$ & $0.565^{* *}$ & $0.569^{* *}$ & $-0.649^{* *}$ \\
\hline 4. ESD & & & & 1 & $0.449^{* *}$ & $0.680^{\text {** }}$ & $0.389^{* *}$ & $-0.401^{* *}$ & -0.1 & -0.043 & 0.265 & 0.205 & $0.357^{* *}$ & 0.274 & $0.314^{* *}$ & $-0.349^{* *}$ \\
\hline 5. EDV & & & & & 1 & $0.644^{* *}$ & $0.654^{* *}$ & -0.294 & -0.249 & -0.117 & -0.058 & 0.236 & $0.542^{* *}$ & $0.542^{* *}$ & $0.545^{*}$ & $-0.626^{* *}$ \\
\hline 6. ESV & & & & & & 1 & 0.24 & $-0.521^{* *}$ & -0.259 & -0.078 & 0.13 & 0.192 & $0.329^{* *}$ & $0.241^{*}$ & $0.280^{*}$ & $-0.447^{* *}$ \\
\hline 7. SV & & & & & & & 1 & 0.053 & -0.217 & -0.142 & -0.152 & 0.172 & $0.412^{* *}$ & $0.471^{* *}$ & $0.444^{* *}$ & $-0.546^{* *}$ \\
\hline 8. EF & & & & & & & & 1 & -0.113 & -0.045 & -0.262 & -0.07 & -0.262 & $-0.327^{* *}$ & -0.273 & 0.055 \\
\hline 9. IVST & & & & & & & & & 1 & $0.728^{* *}$ & 0.154 & -0.061 & $0.522^{* *}$ & $0.384^{* *}$ & $0.545^{* *}$ & $0.829^{* *}$ \\
\hline 10. LVPWT & & & & & & & & & & 1 & 0.059 & 0.097 & $0.637^{* *}$ & $0.437^{* *}$ & $0.623^{* *}$ & $0.770^{* *}$ \\
\hline 11. LA & & & & & & & & & & & 1 & 0.117 & 0.084 & -0.02 & 0.036 & 0.121 \\
\hline 12. Aorta & & & & & & & & & & & & 1 & 0.244 & 0.08 & 0.185 & -0.093 \\
\hline 13. LVMM & & & & & & & & & & & & & 1 & $0.780^{* *}$ & $0.908^{* *}$ & 0.187 \\
\hline 14. LVMMI1 & & & & & & & & & & & & & & 1 & $0.871^{* *}$ & 0.036 \\
\hline 15. LVMMI2 & & & & & & & & & & & & & & & 1 & 0.185 \\
\hline 16. LVRWT & & & & & & & & & & & & & & & & 1 \\
\hline
\end{tabular}

Notes: $*-\mathrm{p}<0.05, * *-\mathrm{p}<0.01$.

Table 8. Correlation between echocardiographic parameters and adipokines in patients with AMI and obesity.

\begin{tabular}{|c|c|c|c|c|c|c|c|c|c|c|c|c|c|c|c|c|}
\hline Indicator & 1 & 2 & 3 & 4 & 5 & 6 & 7 & 8 & 9 & 10 & 11 & 12 & 13 & 14 & 15 & 16 \\
\hline 1. FABP4 & 1 & $-0.487^{*}$ & 0.231 & $0.411^{* *}$ & $0.481^{* *}$ & $0.490^{* *}$ & 0.168 & -0.423 & 0.159 & 0.15 & 0.076 & 0.7 & 0.033 & $0.403^{* *}$ & 0.301 & 0.193 \\
\hline 2. CTRP3 & & 1 & $-0.403^{*}$ & $-0.367^{* *}$ & $-0.326^{*}$ & $-0.453^{* *}$ & -0.01 & 0.493 & -0.073 & -0.026 & 0.201 & -0.108 & -0.142 & $-0.415^{* *}$ & -0.092 & -0.142 \\
\hline 3. EDD & & & 1 & $0.367^{* *}$ & $0.846^{* *}$ & $0.723^{* *}$ & $0.561^{* *}$ & -0.213 & -0.124 & -0.069 & 0.063 & -0.001 & 0.483 & $0.427^{* *}$ & $0.444^{* *}$ & $-0.582^{* *}$ \\
\hline 4. ESD & & & & 1 & $0.728^{* *}$ & $0.794^{* *}$ & 0.186 & $-0.607^{* *}$ & -0.253 & $-0.265^{*}$ & 0.079 & -0.219 & 0.006 & -0.011 & -0.015 & $-0.380^{* *}$ \\
\hline 5. EDV & & & & & 1 & $0.829^{* *}$ & $0.664^{* *}$ & $-0.274^{*}$ & -0.227 & -0.212 & 0.061 & -0.031 & $0.308^{*}$ & $0.266^{*}$ & $0.276^{*}$ & $-0.594^{* *}$ \\
\hline 6. ESV & & & & & & 1 & 0.153 & $-0.672^{* *}$ & -0.196 & -0.119 & 0.157 & -0.149 & $0.301^{*}$ & $0.257^{*}$ & $0.268^{*}$ & $-0.473^{* *}$ \\
\hline 7. SV & & & & & & & 1 & $0.365^{* *}$ & -0.078 & -0.139 & -0.062 & 0.134 & 0.242 & 0.198 & 0.219 & $-0.375^{* *}$ \\
\hline 8. $\mathrm{EF}$ & & & & & & & & 1 & -0.073 & -0.121 & -0.225 & $0.280^{*}$ & -0.241 & -0.176 & -0.206 & 0.007 \\
\hline 9. IVST & & & & & & & & & 1 & $0.742^{*}$ & 0.228 & -0.076 & $0.737^{* *}$ & $0.702^{* *}$ & $0.731^{* *}$ & $0.830^{* *}$ \\
\hline 10. LVPWT & & & & & & & & & & 1 & $0.372^{* *}$ & 0.219 & $0.746^{* *}$ & $0.704^{* *}$ & $0.752^{* *}$ & $0.782^{* *}$ \\
\hline 11. LA & & & & & & & & & & & 1 & 0.22 & $0.337^{* *}$ & $0.290^{*}$ & $0.320^{*}$ & 0.241 \\
\hline 12. Aorta & & & & & & & & & & & & 1 & 0.05 & 0.09 & 0.112 & 0.069 \\
\hline 13. LVMM & & & & & & & & & & & & & 1 & $0.894^{* *}$ & $0.945^{* *}$ & $0.411^{* *}$ \\
\hline 14. LVMMI1 & & & & & & & & & & & & & & 1 & $0.978^{* *}$ & $0.390^{* *}$ \\
\hline 15. LVMMI2 & & & & & & & & & & & & & & & 1 & $0.413^{* *}$ \\
\hline 16. LVRWT & & & & & & & & & & & & & & & & 1 \\
\hline
\end{tabular}

Notes: $*$ - $\mathrm{p}<0.05, * *-\mathrm{p}<0.01$. 


\section{References}

[1] Aleksandrova K, Drogan D, Weikert C, Schulze MB, Fritsche A, Boeing H, et al. Fatty acid-binding protein 4 and risk of type 2 diabetes, myocardial infarction and stroke: a prospective cohort study. The Journal of Clinical Endocrinology \& Metabolism. 2019;104(12):5991-6002. Available from: https://doi.org/10.1210/jc.2019-00477

[2] Furuhashi M. Fatty acid-binding protein 4 in cardiovascular and metabolic diseases. Journal of Atherosclerosis and Thrombosis. 2019;26(3):216-232. Available from: https://doi.org/10.5551/jat.48710

[3] Galderisi M, Cosyns B, Edvardsen T, Cardim N, Delgado V, Di Salvo G, et al. Standardization of adult transthoracic echocardiography reporting in agreement with recent chamber quantification, diastolic function, and heart valve disease recommendations: an expert consensus document of the European Association of Cardiovascular Imaging. European Heart Journal - Cardiovascular Imaging. 2017;18(12):1301-1310. Available from: https://doi.org/10.1093/ehjci/jex244

[4] Granér M, Pentikäinen MO, Nyman K, Siren R, Lundbom J, Hakkarainen A, et al. Cardiac steatosis in patients with dilated cardiomyopathy. Heart. 2014;100(14):1107-1112. Available from: https://doi.org/10.1136/heartjnl-2013304961

[5] Harada T, Sunaga H, Sorimachi H, Yoshida K, Kato T, Kurosawa K, et al. Pathophysiological role of fatty acid-binding protein 4 in Asian patients with heart failure and preserved ejection fraction. ESC Heart Failure. 2020;7(6):4256-4266. Available from: https://doi.org/10.1002/ehf2.13071

[6] Ibanez B, James S, Agewall S, Antunes MJ, BucciarelliDucci C, Bueno H, et al. 2017 ESC Guidelines for the management of acute myocardial infarction in patients presenting with ST-segment elevation. European Heart Journal. 2017;39(2):119-177. Available from: https://doi.org/10.1093/eurheartj/ehx393

[7] Choi K, Hwang S, Hong H, Choi H, Yoo H, Youn $\mathrm{B}-\mathrm{S}$, et al. Implications of $\mathrm{C} 1 \mathrm{q} / \mathrm{TNF}-$ related protein3 (CTRP-3) and progranulin in patients with acute coronary syndrome and stable angina pectoris. Cardiovascular Diabetology. 2014;13(1):14. Available from: https://doi.org/10.1186/1475-2840-13-14

[8] Lamounier-Zepter V, Look C, Schunck W-H, Schlottmann I, Woischwill C, Bornstein SR, et al. Interaction of epoxyeicosatrienoic acids and adipocyte fatty acid-binding protein in the modulation of cardiomyocyte contractility. International Journal of Obesity. 2014;39(5):755-761. Available from: https://doi.org/10.1038/ijo.2014.193
[9] Lang RM, Badano LP, Mor-Avi V, Afilalo J, Armstrong A, Ernande L, et al. Recommendations for cardiac chamber quantification by echocardiography in adults: an update from the American Society of Echocardiography and the European Association of Cardiovascular Imaging. Journal of the American Society of Echocardiography. 2015;28(1):1-39.e14. Available from: https://doi.org/10.1016/j.echo.2014.10.003

[10] Obokata M, Iso T, Ohyama Y, Sunaga H, Kawaguchi $\mathrm{T}$, Matsui $\mathrm{H}$, et al. Early increase in serum fatty acid binding protein 4 levels in patients with acute myocardial infarction. European Heart Journal: Acute Cardiovascular Care. 2016;7(6):561-569. Available from: https://doi.org/10.1177/2048872616683635

[11] Rodríguez-Calvo R, Girona J, Alegret JM, Bosquet A, Ibarretxe $\mathrm{D}$, Masana L. Role of the fatty acid-binding protein 4 in heart failure and cardiovascular disease. Journal of Endocrinology. 2017;233(3):R173-R184. Available from: https://doi.org/10.1530/JOE-17-0031

[12] Durrer Schutz D, Busetto L, Dicker D, Farpour-Lambert N, Pryke R, Toplak H, et al. European practical and patient-centred guidelines for adult obesity management in primary care. Obesity Facts. 2019;12(1):40-66. Available from: https://doi.org/10.1159/000496183

[13] Si Y, Fan W, Sun L. A review of the relationship between CTRP family and coronary artery disease. Current Atherosclerosis Reports. 2020;22(6):22. Available from: https://doi.org/10.1007/s11883-020-00840-0

[14] Tsai H-Y, Wu Y-W, Tseng W-K, Leu H-B, Yin W-H, Lin T-H, et al. Circulating fatty-acid binding-protein 4 levels predict CV events in patients after coronary interventions. Journal of the Formosan Medical Association. 2021;120(1):728-736. Available from: https://doi.org/10.1016/j.jfma.2020.08.007

[15] Von Jeinsen B, Ritzen L, Vietheer J, Unbehaun C, Weferling M, Liebetrau C, et al. The adipokine fattyacid binding protein 4 and cardiac remodeling. Cardiovascular Diabetology. 2020;19(1):117. Available from: https://doi.org/10.1186/s12933-020-01080-X

[16] Wang S, Ling Y, Liang W, Shen L. Association of serum C1q/TNF-related protein-3 (CTRP-3) in patients with coronary artery disease. BMC Cardiovascular Disorders. 2017;17(1):210. Available from: https://doi.org/10.1186/s12872-017-0646-7

[17] Wu D, Lei H, Wang J-Y, Zhang C-L, Feng H, Fu F-Y, et al. CTRP3 attenuates post-infarct cardiac fibrosis by targeting Smad3 activation and inhibiting myofibroblast differentiation. Journal of Molecular Medicine. 2015;93(12):1311-1325. Available from: https://doi.org/10.1007/s00109-015-1309-8 
[18] Yi W, Sun Y, Yuan Y, Lau WB, Zheng Q, Wang $\mathrm{X}$, et al. $\mathrm{C} 1 \mathrm{q} /$ tumor necrosis factor-related protein3 , a newly identified adipokine, is a novel antiapoptotic, proangiogenic, and cardioprotective molecule in the ischemic mouse heart. Circulation. 2012;125(25):3159-3169. Available from: https://doi.org/10.1161/CIRCULATIONAHA.112.099937

[19] Yuan Y-P, Ma Z-G, Zhang X, Xu S-C, Zeng X-F, Yang $Z$, et al. CTRP3 protected against doxorubicininduced cardiac dysfunction, inflammation and cell death via activation of Sirt1. Journal of Molecular and Cellular Cardiology. 2018;114:38-47. Available from: https://doi.org/10.1016/j.yjmcc.2017.10.008
[20] Zhang Z, Zhu L, Feng P, Tan Y, Zhang B, Gao $\mathrm{E}$, et al. $\mathrm{C} 1 \mathrm{q} /$ tumor necrosis factor-related protein-3engineered mesenchymal stromal cells attenuate cardiac impairment in mice with myocardial infarction. Cell Death \& Disease. 2019;10(7):530. Available from: https://doi.org/10.1038/s41419-019-1760-5

Received: $2021-04-28$

Revised: 2021-07-13

Accepted: 2021-08-11 